\title{
The Rainwater Memorial Calibration Facility for X-Ray Optics
}

\author{
Nicolai F. Brejnholt, ${ }^{1}$ Finn E. Christensen, ${ }^{1}$ Charles J. Hailey, ${ }^{2}$ Nicolas M. Barrière, ${ }^{3}$ \\ William W. Craig, ${ }^{3}$ Brian Grefenstette, ${ }^{4}$ Jason Koglin, ${ }^{2}$ Kristin Kruse Madsen, ${ }^{4}$ \\ Julia K. Vogel, ${ }^{5}$ Hongjun An, ${ }^{2}$ Kenneth Blaedel, ${ }^{2}$ Josh Brown, ${ }^{2}$ Todd Decker, ${ }^{5}$ Zeshan Haider, ${ }^{2}$ \\ Anders Clemen Jakobsen, ${ }^{1}$ Carsten P. Cooper-Jensen, ${ }^{1}$ Kaya Mori, ${ }^{2}$ Melania Nynka, ${ }^{2}$ \\ Michael J. Pivovaroff,, ${ }^{5}$ Clio Sleator, ${ }^{2}$ Dennis Stefanik,, ${ }^{2}$ Marcela Stern, ${ }^{2}$ Gordon Tajiri, ${ }^{2}$ \\ Douglas Thornhill, ${ }^{2}$ and Jeremy S. Cushman ${ }^{2}$
}

\author{
${ }^{1}$ DTU Space, Technical University of Denmark, DK-2100 Copenhagen, Denmark \\ ${ }^{2}$ Columbia University Astrophysics Laboratory, New York, NY 10027, USA \\ ${ }^{3}$ Space Sciences Laboratory, University of California, Berkeley, CA 94720, USA \\ ${ }^{4}$ Space Radiation Laboratory, California Institute of Technology, Pasadena, CA 91125, USA \\ ${ }^{5}$ Physics Division, Physical and Life Science Directorate, LLNL, Livermore, CA 94550, USA
}

Correspondence should be addressed to Nicolai F. Brejnholt, nicolai@space.dtu.dk

Received 11 February 2011; Accepted 30 June 2011

Academic Editor: Lars Furenlid

Copyright (C) 2011 Nicolai F. Brejnholt et al. This is an open access article distributed under the Creative Commons Attribution License, which permits unrestricted use, distribution, and reproduction in any medium, provided the original work is properly cited.

The Nuclear Spectroscopic Telescope ARray (NuSTAR) is a NASA Small Explorer mission that will carry the first focusing hard X-ray $(5-80 \mathrm{keV})$ telescope to orbit. The ground calibration of the optics posed a challenge as the need to suppress finite source distance effects over the full optic and the energy range of interest were unique requirements not met by any existing facility. In this paper we present the requirements for the NuSTAR optics ground calibration, and how the Rainwater Memorial Calibration Facility, RaMCaF, is designed to meet the calibration requirements. The nearly $175 \mathrm{~m}$ long beamline sports a $48 \mathrm{~cm}$ diameter $5-$ $100 \mathrm{keV}$ X-ray beam and is capable of carrying out detailed studies of large diameter optic elements, such as the NuSTAR optics, as well as flat multilayer-coated Silicon wafers.

\section{Introduction}

The Nuclear Spectroscopic Telescope Array (NuSTAR), a NASA SMall EXplorer (SMEX) satellite mission, will herald a new age for hard X-ray astronomy.

The two identical optics onboard NuSTAR will provide the scientific community with significant improvements in sensitivity compared to present-day instruments (cf. Figure 1), and be the first graded multilayer-coated space Xray telescope (originally suggested by Christensen et al. [1]) operating up to $80 \mathrm{keV}$.

Each optic is comprised of 2340 pieces of multilayercoated glass substrates $225 \mathrm{~mm}$ long and $0.21 \mathrm{~mm}$ thick, distributed over 133 concentric shells. Individual substrates span $30^{\circ}$ (layer 69-133) or $60^{\circ}$ (layer 1-68) and have radii ranging from $54.4 \mathrm{~mm}$ to $191 \mathrm{~mm}$. The coatings, utilizing
Pt/C (layer 1-89) and W/Si (layer 90-133), are optimized for energies between 5 and $80 \mathrm{keV}$.

The thermally slumped glass, assembled in a conical approximation to Wolter-I geometry, has low roughness $(<5 \AA)$ and will be mounted on graphite spacers precisionmachined to give grazing incidence angles from 0.077 to $0.27^{\circ}$. These angles allow the $37.5 \mathrm{~kg}$ heavy optics to focus photons at a focal length of $10.15 \mathrm{~m}$ with an on-axis angular resolution of $45^{\prime \prime}$ (HPD).

The NuSTAR telescope parameters are summarized in Table 1. For a more complete explanation of the NuSTAR mission, science, and optics, see Harrison et al. [2] and Hailey et al. [3] .

NuSTAR will be the first focusing hard X-ray telescope to undergo extensive calibration. Since most cosmic hard Xray sources are time variable, and since there will be few 


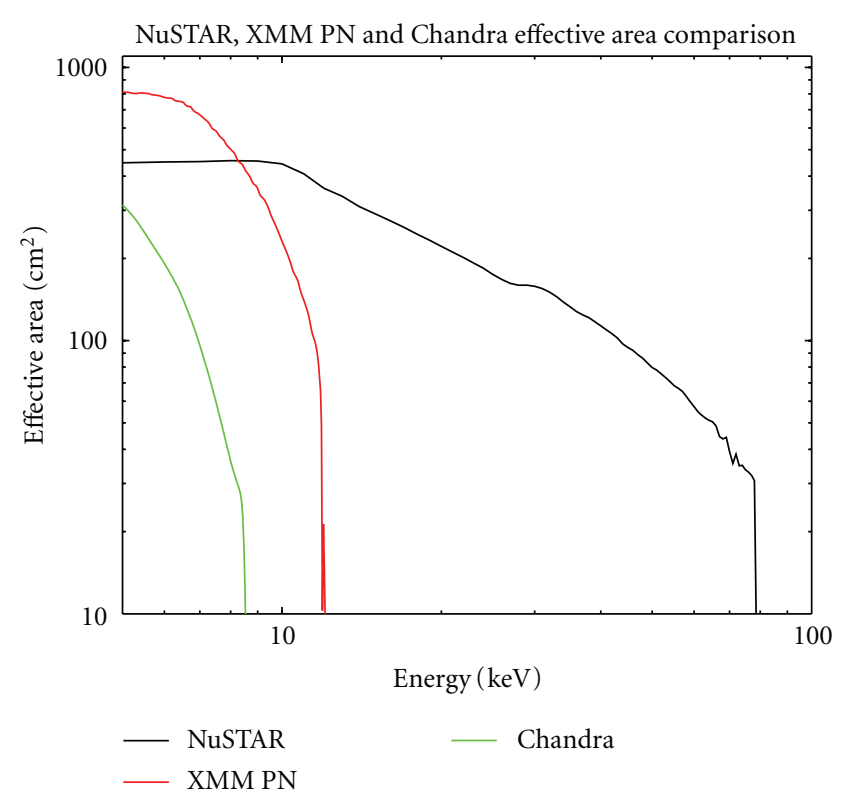

Figure 1: Comparison between the effective areas of the ongoing $\mathrm{XMM}$ and Chandra missions to the expected effective area of NuSTAR [2].

TABLE 1: Overview of NuSTAR telescope parameters.

\begin{tabular}{lc}
\hline \multicolumn{2}{c}{ NuSTAR telescope parameters } \\
\hline Focal length & $10.15 \mathrm{~m}$ \\
Shell radii & $54.4-191 \mathrm{~mm}$ \\
Graze angles & $0.077-0.27^{\circ}$ \\
Shell length & $225 \mathrm{~mm}$ \\
Shell thickness & $0.21 \mathrm{~mm}$ \\
Total shells per module & 133 \\
Multilayer (Layer 1-89) & $\mathrm{Pt} / \mathrm{C}$ \\
Multilayer (Layer 90-133) & $\mathrm{W} / \mathrm{Si}$ \\
Energy band & $5-80 \mathrm{keV}$ \\
Angular resolution & $45^{\prime \prime}(\mathrm{HPD})$ \\
Mass per optic & $37.5 \mathrm{~kg}$ \\
\hline
\end{tabular}

hard X-ray instruments available contemporaneously onorbit for cross calibrations, careful attention to execution of the ground calibration is required. There are several unique challenges associated with the low graze angles, multilayer coatings, and high X-ray energies. Relative to those with simple metal coatings, multilayer optics have more complicated response as a function of incident angle and energy.

No existing long-beam X-ray facilities extend to high enough energy and are available for a sufficient time periods to be utilized to develop and execute the requisite NuSTAR optics calibration. In addition, frequent access to an intense collimated X-ray beam enables routine monitoring of multilayer-coated witness substrate reflectance for process control during the mirror fabrication, and for rapid evaluation of prototype and engineering models. For these reasons,

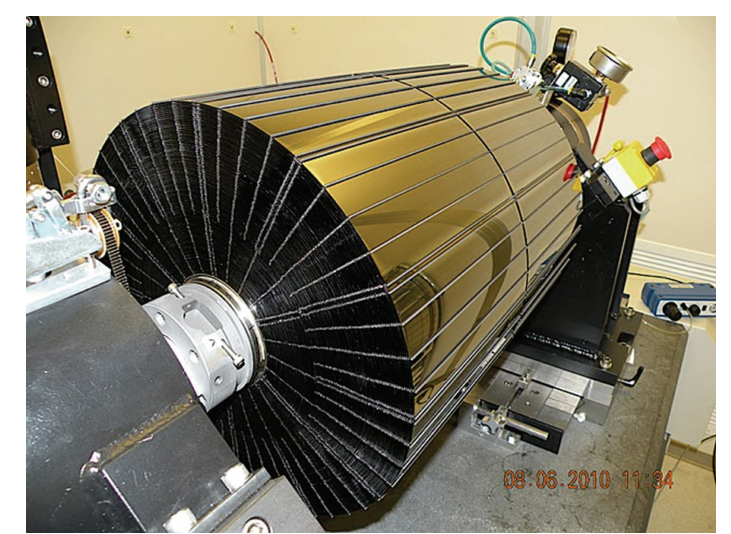

FIGURE 2: Picture of FM0 mounted on the assembly machine on the day of completion in August 2010. Picture courtesy of Todd Decker.

the Rainwater Memorial Calibration Facility, RaMCaF was developed.

The availability of a $170 \mathrm{~m}$ long, underground tunnel, neighboring the NuSTAR optics' assembly room at Nevis Laboratories in New York, allowed construction of the Rainwater Memorial Calibration Facility (RaMCaF, see end note for historical details) to be done on a minimum budget and within the short timeframe allotted from its conception in late 2008 to its scheduled use in January 2010.

The first optics module, FM0, which is a full-scale qualification model, pictured in Figure 2, was completed in August 2010 and was calibrated in November 2010 prior to environmental tests, and again in January 2011 following environmental tests. Flight modules FM1 and FM2 are scheduled to be completed in March 2011.

This paper describes the optics ground calibration requirements, the derived facility requirements, and finally the facility itself and the calibration approach.

\section{Optics Calibration Requirements}

The NuSTAR calibration approach calls for an absolute calibration of response of the flight detectors, and calibration of the relative effective area of the optics as a function of area and off-axis angle. The overall absolute telescope response calibration requirements will be met using on-orbit observations of the Crab. Although absolute throughput measurements of the optics will be made as a part of the calibration program, stringent requirements are levied only on the relative optics response determination. The relative effective area $A_{\text {eff }}$ must be determined to within three percent in the energy band 5-60 keV (bins of $2 \mathrm{keV}$ ) and two percent between 60 and $80 \mathrm{keV}$ (bins of $1 \mathrm{keV}$ ). The point spread function (PSF) calibration to a level required for science data extraction will be performed on-orbit using bright point sources (e.g., Cyg X-1) and will be measured to about $5 \%$ in order to verify that the optics meet resolution requirements. Both the PSF and relative $A_{\text {eff }}$ must be investigated on-axis and at two off-axis angles. The calibration of each flight 
TABLE 2: Overview of NuSTAR ground calibration requirements.

\begin{tabular}{lc}
\hline \multicolumn{2}{c}{ NuSTAR calibration requirements } \\
\hline$\Delta A_{\text {eff }}(5-60 \mathrm{keV})$ & $3 \%$ \\
$\Delta A_{\text {eff }}(60-80 \mathrm{keV})$ & $2 \%$ \\
Bin size $(5-60 \mathrm{keV})$ & $2 \mathrm{keV}$ \\
Bin size $(60-80 \mathrm{keV})$ & $1 \mathrm{keV}$ \\
$\Delta \theta_{\text {PSF }}(6-80 \mathrm{keV})$ & $5 \%$ \\
$T_{\text {calibration }}$ & $10^{6}$ seconds \\
\hline
\end{tabular}

module is allotted a total of $10^{6}$ seconds $(<12$ days $)$ in the project schedule.

The main difficulties in achieving the goals tabulated in Table 2 within this time frame herald from the finite source distance available (17 times the focal length of the telescope) for on-ground measurements and the attenuation of low energy photons in air. The approach to addressing these issues is described below.

\section{Calibration Facility Requirements}

Accurate calibration of the effective area and, to a lesser degree, determining the PSF in the focal plane for requirements verification drive all facility requirements, along with features inherent to the optics, such as focal length and energy band.

Ideally a source located at infinity would fully flood the entrance aperture of the optic with a parallel beam of Xrays, such as will be the case in space. At RaMCaF, this is approximated by placing the X-ray source at the far end of the tunnel. The finite distance $S$ means that the optic-focal plane distance $f_{S}$, at RaMCaF, will be slightly longer than the nominal focal length $f$, as given by the Lens-Maker equation.

$$
\frac{1}{f_{S}}=\frac{1}{f}-\frac{1}{S}
$$

NuSTAR flight requirements state that $f$, and by extension $f_{S}$, must be established with $\pm 10 \mathrm{~mm}$ accuracy to reduce broadening of the PSF from being out-of-focus. This translates into a rather lax requirement on knowledge of $S$ at RaMCaF. However, to reserve $\Delta f_{S}$ for optics and detector misplacement, $S$ is required to be known within $\pm 10 \mathrm{~cm}$.

The source-optic distance means that the $\mathrm{X}$-rays, especially at lower energies, are attenuated heavily, prompting the requirement for a vacuum pipeline. The modest budget and timeframe prevented constructing the entire facility in vacuum, but the maximum allowable total length of ambient air from source-focal plane was set to $2 \mathrm{~m}$ allocated entirely for beamline hardware. The remaining length is required to be pumped down below 1 Torr, such that attenuation at $6 \mathrm{keV}$ is dominated by the air gap.

The pipeline and all other components of the facility must be part of one coordinate system, which runs along the chief X-ray axis as defined by three fixed points in the facility. First point is defined by the X-ray source, while the second point is located just prior to the front end of the optics. Third point is an optical scope located beyond $f_{S}$ and pointed along
TABLE 3: Required knowledge of source-optic $(\Delta S)$ and optic-focal plane $\left(\Delta f_{S}\right)$ distance as well as maximum allowable air gap and pipeline vacuum level at RaMCaF.

\begin{tabular}{lc}
\hline \multicolumn{2}{c}{ Facility requirements } \\
\hline$\Delta f_{S}$ & $\pm 10 \mathrm{~mm}$ \\
$\Delta S$ & $\pm 10 \mathrm{~cm}$ \\
Air gap & $\leq 2 \mathrm{~m}$ \\
Vacuum level & $<1$ Torr \\
\hline
\end{tabular}

the chief X-ray axis, allowing all components between the Xray source and the detector to be optically aligned to the chief $\mathrm{X}$-ray axis.

The optic mounts at the origin of the coordinate system. To facilitate an accurate and reproducible alignment, several well-defined motions of the optic mount are required. These are summarized in Table 4 along with the range and precision required and motions necessary to carry out the actual calibration of the optic, as discussed later. The facility coordinate system is also shown in the table.

$A_{\text {eff }}$ will be determined through $I / I_{0}$ measurements requiring a stable $\mathrm{X}$-ray beam and detector electronics. To check the stability of the X-ray source output and the influence of pressure, temperature, and humidity changes, a dedicated low energy X-ray beam monitor is also required. To quantify observed changes in the low energy spectrum, the ambient pressure, temperature and humidity, as well as the pipe pressure must be monitored.

\section{Calibration Facility}

4.1. Beam Line Layout. The full length of the beam line is $174 \mathrm{~m}$ from the X-ray tube to the focal plane of the NuSTAR optic. Referring to Figure 3, the X-ray tube (a) is situated inside the source room along with a filter unit (b), collimator (c), and a Kapton window (d) to the tunnel pipeline. The pipeline leads through the tunnel out into the high bay area where the calibration room and detector room is located.

The tunnel pipeline has five rotary vane pumps connected with a total pumping capacity of $130 \mathrm{~m}^{3} / \mathrm{h}$ bringing the roughly $14 \mathrm{~m}^{3}$ volume down to 1 Torr in less than half a day. The detector pipeline with a volume of roughly $2 \mathrm{~m}^{3}$ is pumped down to 1 Torr in a few hours with a single $28 \mathrm{~m}^{3} / \mathrm{h}$ pump. The pipeline is mainly constructed from Schedule 40 PVC pipes and range in diameter from $10 \mathrm{~cm}$ (near source) to $50 \mathrm{~cm}$ (near optic and entire detector pipeline). The high outgassing rate of PVC compared to f.ex. stainless steel was justified by the relatively low vacuum requirement and costeffectiveness.

The calibration room is a roughly $5 \mathrm{~m}^{2}$ class 1000 clean room. The beam enters through one of the three customfabricated Kevlar reinforced Mylar windows (e, j, and k). The Kevlar backing not only reduces the deflection of the Mylar, but safeguards the optic in the event of a Mylar rupture, as the vacuum bleeds slowly up to ambient through the Kevlar weave. The laminate is illustrated in Figure 4.

The room has facilities for installing various beam apertures ( $f$ and $h$ ), utilized during the optic calibration 
TABle 4: Motion requirements for RaMCaF. The facility has a right-handed Cartesian coordinate system with the chief X-ray axis defined as being the $z$-axis. Translations occur in $X$ while roll is defined as a rotation around the $z$-axis, yaw around the $y$-axis and pitch around the $x$-axis. An optic will be perfectly aligned when its center of rotation in $x, y$, and $z$ is in the origin. $z$ values are positive in the direction of the focal plane.

\begin{tabular}{|c|c|c|c|c|}
\hline & equireme & & $y$ & \\
\hline Motion & Range & Precision & & \\
\hline Translate (optic) & $300 \mathrm{~mm}$ & $\pm 0.025 \mathrm{~mm}$ & & $z$ \\
\hline Translate (detector) & $200 \mathrm{~mm}$ & $\pm 0.025 \mathrm{~mm}$ & & \\
\hline Roll & $360^{\circ}$ & $\pm 0.1^{\circ}$ & & \\
\hline Yaw & $> \pm 1^{\circ}$ & $\pm 0.00025^{\circ}$ & & \\
\hline Pitch & $> \pm 0.5^{\circ}$ & $\pm 0.0015^{\circ}$ & & \\
\hline
\end{tabular}

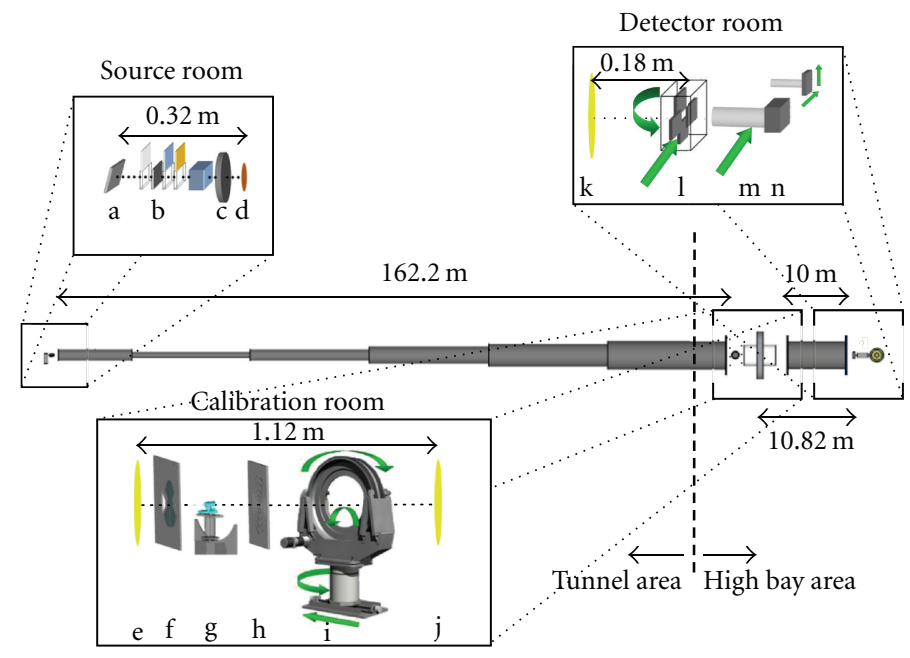

Figure 3: Facility overview with source room connected to calibration room by $162.2 \mathrm{~m}$ long tunnel pipe line and calibration room connected to detector room by $10 \mathrm{~m}$ detector pipe line. The individual components are (a) high power X-ray tube (b) thin four-film filter unit, and thick (up to $50 \mathrm{~mm}$ ) attenuator. The filter unit is remote controlled, while the attenuator block must be inserted and removed manually. (c) Collimator plate with $12 \mathrm{~mm}$ aperture, (d) $22 \mathrm{~mm}$ diameter $75 \mu \mathrm{m}$ thick Kapton vacuum window, (e) $48 \mathrm{~cm}$ diameter Kevlarreinforced Mylar vacuum window, (f) coarse aperture plate, (g) autocollimator, (h) fine aperture plate, (i) calibration bench for mounting optic. Available movements are translation, roll, yaw, and pitch indicated by green arrows ( $\mathrm{j}$ and $\mathrm{k}$ ) $48 \mathrm{~cm}$ Kevlar-reinforced Mylar vacuum windows. (l) Four-blade slit unit. Each blade is $4 \mathrm{~mm}$ thick Tungsten. Maximum aperture size is $30 \times 30 \mathrm{~mm}^{2}$ and the entire unit can be translated and rotated in yaw $(\mathrm{m}) \mathrm{X}$-ray detector. Detector can be translated (n) Beam monitor. Beam monitor can be translated in both $X$ and $Y$ to facilitate unobstructed view during calibration.

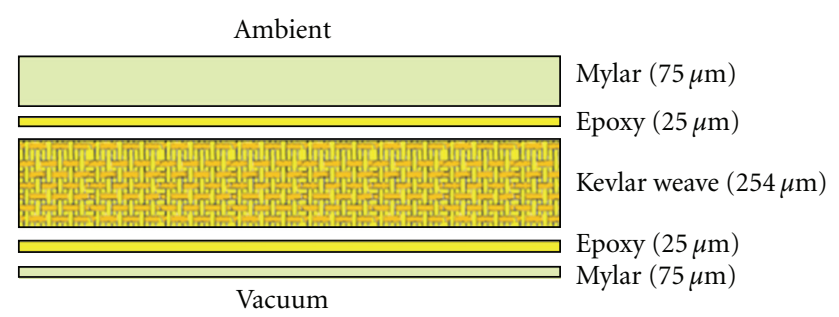

Figure 4: Kevlar weave sandwiched between two thin Mylar sheets was bonded together in a flat vacuum mold with a two-part epoxy resin to produce several $48 \mathrm{~cm}$ diameter Kevlar-reinforced Mylar vacuum windows.

discussed later, as well as an autocollimator setup $(\mathrm{g})$ which provides knowledge of the angular movements of the optic during calibration. It further houses a calibration bench custom-built for testing focusing X-ray optics (i). The bench was previously used to calibrate SODART [4] and HEFT [5].

The source-optic distance $S$ is $163.1 \mathrm{~m}$, resulting in $f_{s}$ being $10.82 \mathrm{~m}$ for the NuSTAR optics. This governs the length of the facility past the optics. The detector pipeline connects the calibration room to the detector room which houses a slit unit (l), as well as the focal plane detectors (m).

Finally a low energy Silicon detector is mounted in the focal plane to act as a beam monitor (n). Due to the geometry of the optic fixture and the calibration approach (see below) the placement of this unit was not trivial and requires the monitor to be mounted on an XY stage to prevent occasional obscurations.

The X-ray source, filters, and all motions in the facility are controlled from a control room that overlooks the optics and detector rooms. All data acquisition and environmental logging also takes place from the control room. 


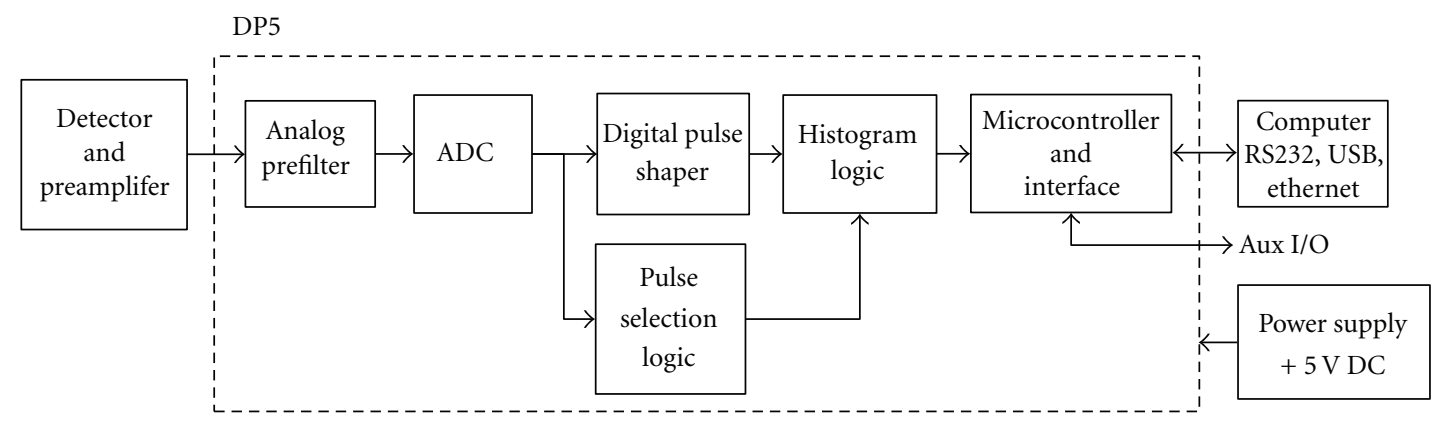

FIGURE 5: Block diagram indicating the DP5 setup. The block diagram is taken from the DP5 product sheet.

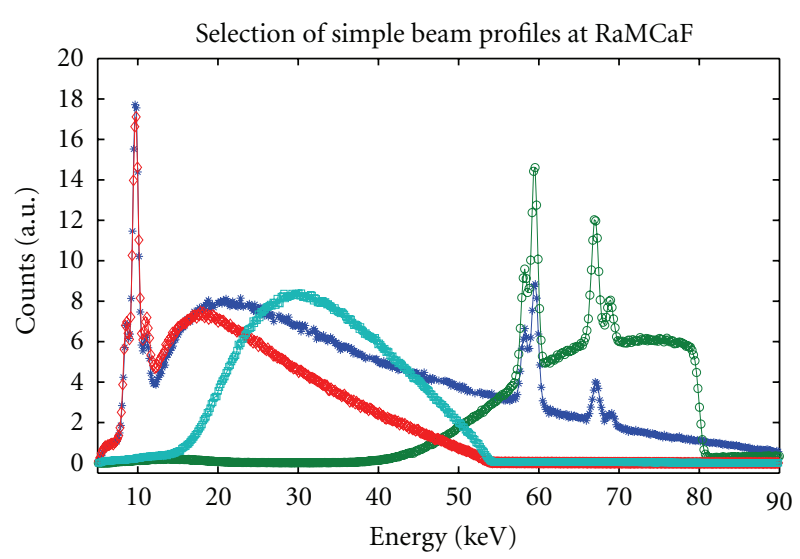

$\rightarrow$ No filter, $100 \mathrm{kV}, 1 \mathrm{~mA}$

$\rightarrow 0.25 \mathrm{~mm} \mathrm{Au}, 100 \mathrm{kV}, 15 \mathrm{~mA}$

$\rightarrow$ No filter, $60 \mathrm{kV}, 1.5 \mathrm{~mA}$

$-2.56 \mathrm{~mm} \mathrm{Al}, 60 \mathrm{kV}, 5 \mathrm{~mA}$

FIGURE 6: Selection of source spectra obtained at near maximum rate on Ge detector. Simple filtering enables higher rates in selected bands to be achieved. This allows for more detailed and less time-consuming investigation of the high energy response of the telescope.

The entire tunnel area and the two rooms situated in the high bay, as well as the exposed detector pipe line, have been shielded to allow nonradiation workers access to the surrounding areas while extensive environmental and security monitoring has been implemented at RaMCaF. The purpose of this is threefold. First off the facility operates a powerful X-ray source and must ensure the safety and wellbeing of its personnel, which is done through shielding and multiple layers of safety interlocks, each of which terminates generation of X-rays, if tripped, as well as video and access monitoring.

Secondly, the facility must ensure the safety and wellbeing of the NuSTAR flight modules during calibration. Exposure to dirt, high humidity, or temperature, are all factors that may lead to degraded performance of the optic. These exposures are sought limited in the temperature and humidity regulated class 1000 clean room. Even so they are all monitored to ensure that any accidental exposure is on record. The room is further access restricted to authorized personnel through RFID cards.
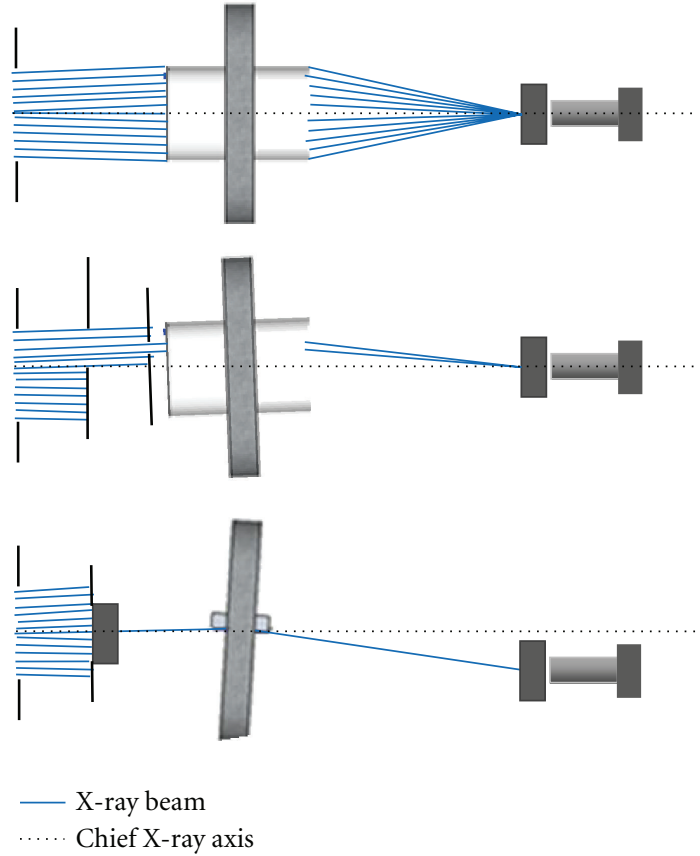

FIGURE 7: Sketch of full flood illumination (top) and subaperture testing (middle) of the optic as well as flat witness sample test setup (bottom). (Top) Full flood illumination has no limitations on the beam before the focusing optic is hit, but a detector slit is located at the focus before the detector. Alignment happens first through optical means then using X-rays and full flood illumination. (Middle) Subaperture testing involves a coarse aperture plate followed by a fine aperture plate which selects a subgroup of layers for investigation. To achieve a pseudoparallel beam, the optic is rotated in yaw. (Bottom) The aperture plates are replaced with an optic slit (identical to detector slit) and an extremely flat vacuum chuck is mounted in place of the optic fixture. Flat witness samples can now be mounted and tested with a grazing incidence white pencil beam.

Finally it is imperative that any discrepancies in the calibration results can be tracked down through knowledge of the conditions under which the calibration was carried out, specifically the pressure in the pipeline, the ambient pressure, temperature, and humidity, all of which influence the beam spectrum and intensity to varying degree.

All monitored data is displayed live in the control room and logged to a web server to allow collaborators easy access. 


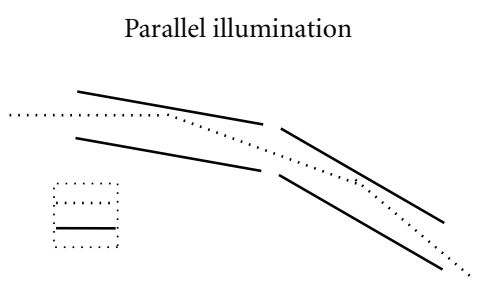

(a)

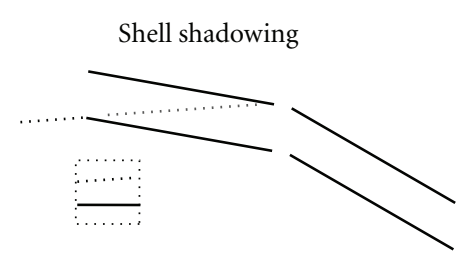

(c)

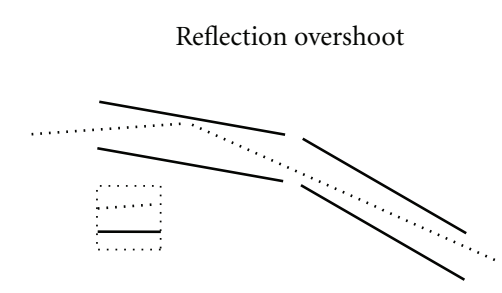

(b)

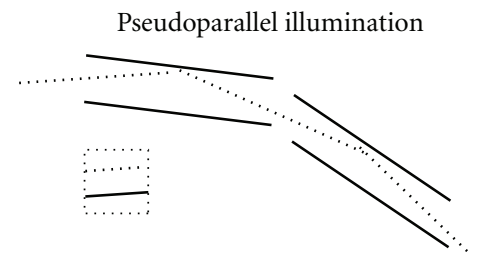

(d)

FIgURE 8: Sketch of finite source distance implications. The dotted box contains lines parallel to the optic axis (full line) and the X-ray axis (dotted line) in each of the four cases. (a) Ideal situation with beam parallel to optic axis. (b) Reflection overshoot occurs when beam divergence causes reflected beam to miss second mirror. (c) Shell shadowing may also occur, but the distance between mirror shells in the NuSTAR optic means it does not contribute here. (d) Pseudoparallel illumination compensates for the beam divergence by adjusting the yaw of the optic. The divergence of the beam in this approach is determined by the radial size of the subgroup under investigation rather than the radial location of the subgroup.

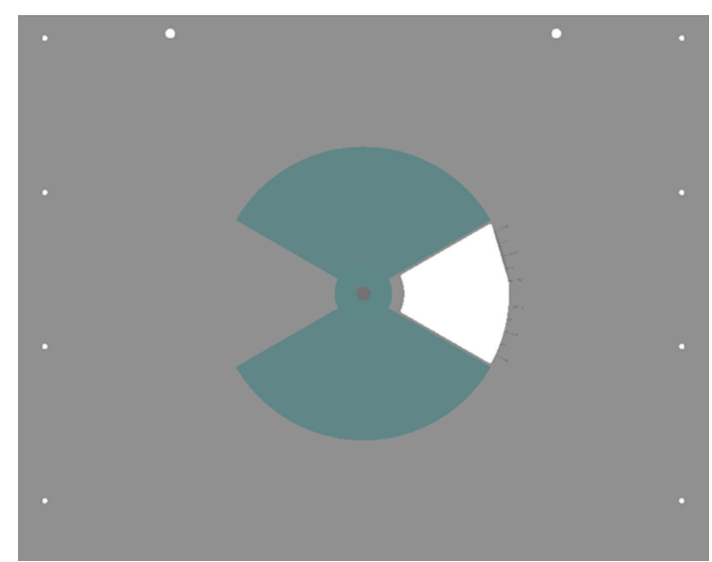

(a)

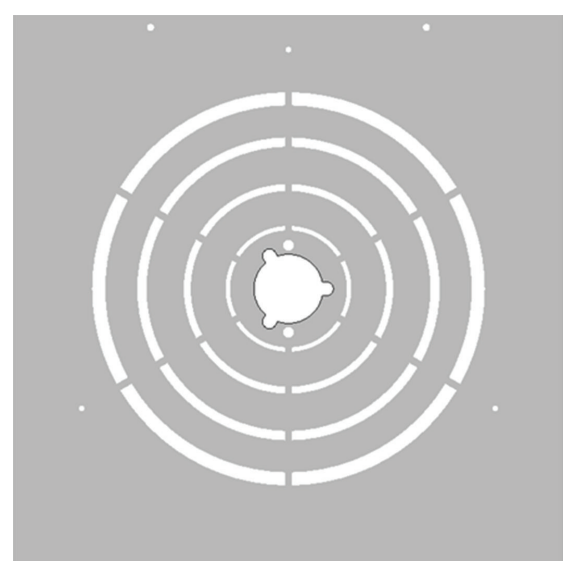

(b)

Figure 9: Front view of coarse aperture plate (a) and fine aperture plate (b). The plates consist of 4 mm of lead sandwiched with Aluminum. (a) Bowtie is shown which can be used to select between $15^{\circ}$ and $60^{\circ}$ of the azimuthal range (b) Fine aperture plate to select the radial range(s) and more finely define the azimuthal extent to sextants. The plate mounts directly to the optic.

4.2. Data Acquisition System. The workhorse in the RaMCaF DAQ setup is a state-of-the-art Amptek DP5 [6]. This low power digital pulse processor replaces both shaping amplifier and multichannel analyzer (MCA) and provides for Ethernet communication. The DP5 supports both reset and feedback preamplifiers of either polarity (although it must be customized through the addition of a resistor for resistor feedback preamplifiers). Pile-up events separated by more than the fast channel resolving time of $120 \mathrm{~ns}$ are rejected. The dead-time per pulse is limited to 1.05 times the peaking time with no additional conversion time and is corrected based on the fast channel event rate. Figure 5 shows a block diagram of a DP5 in a complete system. At RaMCaF, the Ethernet communication allows LabVIEW [7] to start and stop acquisition as well as read out or clear the DP5 histogram memory and set up analog gain and pulse shaping time.

4.3. Calibration Detectors. Four detectors are available for flight module ground calibration at RaMCaF.

To fulfill requirements on effective area as a function of energy, a coaxial Germanium $(\mathrm{Ge})$ detector is used for energies above $12 \mathrm{keV}$, while a Silicon Drift Detector (SDD) is used down to $5 \mathrm{keV}$. The Ge detector is an Ortec GammaX HPGe (GMX25P4-70) with built-in preamplifier and high voltage filter [11]. The SDD is a prototype detector developed by the Centre d'Etude Spatiale des Rayonnements (CESR) based on a large area chip by PNDetector GmbH [12]. 
TABle 5: RaMCaF detector specifications. Quoted energy resolution is FWHM. Note that the image area of the EMCCD detector can be tripled using a $3: 1$ taper also available.

\begin{tabular}{|c|c|c|c|}
\hline Ge detector & & Silicon drift detector & \\
\hline Calibration bandpass & $12-80 \mathrm{keV}$ & Calibration bandpass & $5-25 \mathrm{keV}$ \\
\hline Energy resolution@22 keV & $715 \mathrm{eV}$ & Energy resolution@ @ $5.9 \mathrm{keV}$ & $135 \mathrm{eV}$ \\
\hline Crystal diameter & $58 \mathrm{~mm}$ & Crystal area & $100 \mathrm{~mm}^{2}$ \\
\hline Crystal thickness & $48 \mathrm{~mm}$ & Crystal thickness & $450 \mu \mathrm{m}$ \\
\hline Window thickness & $0.5 \mathrm{~mm}(\mathrm{Be})$ & Window thickness & $25 \mu \mathrm{m}$ \\
\hline EMCCD detector & & Beam monitor & \\
\hline Calibration bandpass & Imaging & Calibration bandpass & N/A \\
\hline Pixel size & $16 \times 16 \mu \mathrm{m}^{2}$ & Energy resolution@ @ $5.9 \mathrm{keV}$ & $149 \mathrm{eV}$ \\
\hline Image area & $8.2 \times 8.2 \mathrm{~mm}^{2}$ & Crystal area & $5 \times 5 \mathrm{~mm}^{2}$ \\
\hline Max readout rate & $10 \mathrm{MHz}$ & Crystal thickness & $300 \mu \mathrm{m}$ \\
\hline Frame rate & $35-549 \mathrm{fps}$ & Window thickness & $25.4 \mu \mathrm{m}$ \\
\hline
\end{tabular}

TABle 6: RaMCaF X-ray source specifications. The information derives from the Comet product sheet.

\begin{tabular}{lc}
\hline \multicolumn{2}{c}{ Comet X-ray source } \\
\hline Nominal X-ray tube voltage & $100 \mathrm{kV}$ \\
Continuous rating & $3 \mathrm{~kW}$ \\
Tube current at nominal voltage, max. & $30 \mathrm{~mA}$ \\
Focal spot acc. EN 12543 (diameter at $1 \mathrm{~kW})$ & $7.5 \mathrm{~mm}$ \\
Inherent filtration & $0.8 \pm 0.1 \mathrm{~mm}$ \\
Target material & Beryllium \\
Target angle & Tungsten \\
High voltage ripple & $30^{\circ}$ \\
Induced temperature drift (voltage) & $5 \mathrm{~V} / \mathrm{mA}$ (min. $20 \mathrm{~V}$ ) \\
Induced temperature drift (current) & $80 \mathrm{ppm} /{ }^{\circ} \mathrm{C}$ \\
Voltage accuracy & $50 \mathrm{ppm} /{ }^{\circ} \mathrm{C}$ \\
Current accuracy & $\pm 1 \%$ of output value \\
\hline
\end{tabular}

A 2D detector is also available at the facility for alignment and PSF investigation purposes. The detector is a prototype model developed in collaboration between Andor Technology and RMD Inc. [13]. The design is a novel electron multiplying CCD (EMCCD) which is coupled to a thin microcolumnar scintillator film (CsI ( $\mathrm{Tl})$ ) via a fiber optic taper.

Finally an Amptek XR-100CR high performance X-ray detector [14], utilizing a Silicon PIN photodiode, is installed in the focal plane to monitor the stability of the beam.

Table 5 describes a number of characteristics of the four detectors.

4.4. X-Ray Source. The X-ray source utilized at RaMCaF is a potent Comet XRP-160 high voltage generator powering an MXR-161 X-ray tube with a Tungsten target [15]. The tube is limited to a maximum high voltage of $100 \mathrm{kV}$ and a current of $30 \mathrm{~mA}$. It has a very low high voltage ripple and temperature drift as well as accurate and repeatable settings. Further details are available in Table 6.
It was recognized early on that a high power tube would be required to provide sufficient statistics especially at low energies due to absorption and scattering along the beam line. However, the potency of the Comet and the large effective area of the optic means that most measurements at high energy will be acquisition limited at low current setting, with the bulk of photons having energy between $10 \mathrm{keV}$ and $40 \mathrm{keV}$. To improve on the effective area measurements at high energy, a filter unit allows simple low energy, and more elaborate $K$-edge, filtering to be carried out. Figure 6 shows a source spectrum with and without filtering and with various source settings. Only the low energy filtering is required for calibration purposes.

4.5. Calibration Approach. Once the optic has been installed in the calibration bench, the detector will be temporarily removed and an optical scope, anchored behind the detector room, will be used as an autocollimator to align the optic to the chief X-ray axis. Once this initial alignment has been carried out, the detector is replaced and the calibration room autocollimator (see Figure 3) is nulled to the aligned optic. Throughout the calibration process, this autocollimator will provide arc second knowledge of the pitch and yaw of the optic.

The X-ray alignment of the optic is naturally quite critical and will be carried out using full flood geometry, as shown in Figure 7(top). The alignment procedure will consist of scanning in pitch and yaw in an iterative manner until the maximum throughput is found. Moving the optic through roll validates that the chief X-ray axis is aligned to the mechanical axis of the optic.

One caveat in this approach is that the finite source distance results in an incomplete sampling of the optic due to the beam divergence as illustrated in Figure 8. To remedy this, aperture plates are installed in front of the optic as illustrated in Figure 7(middle). The coarse aperture plate allows selecting between $15^{\circ}$ and $60^{\circ}$ of the optic for exposure, while the fine aperture plate picks out a subgroup of radial layers within the selected azimuthal range. Once a fine aperture plate has been installed, the optic yaw is offset 


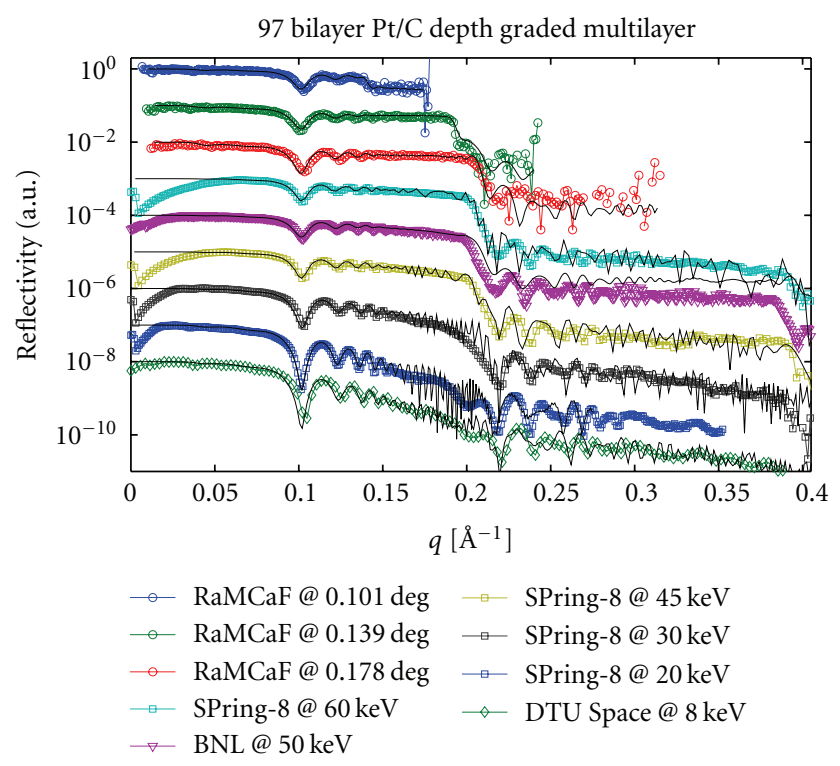

Figure 10: Comparison of data taken at RaMCaF with $100 \mathrm{kV}$ tube voltage to synchrotron data from SPring-8 [8] and the X17B1 beamline at Brookhaven National Labs as well as a rotating anode setup at DTU Space [9]. The data was fit in IMD [10] using the same multilayer recipe in each case. The fit is shown as black lines. Each data set is off-set by an order of magnitude from previous data set.

by an amount corresponding to the divergence of the beam (at the selected radii) yielding a pseudoparallel beam.

A number of fine aperture plates exist in order to select each of the twenty subgroups that make up the full optic. The selections are dubbed "subgroups" as the optic design has ten separate multilayer "groups" each of which have been divided into two subgroups. See [16] for additional details on the individual multilayer groups.

A frontal view of the coarse aperture plate and an example of a fine aperture plate are shown in Figure 9. The main calibration effort consists of measuring each of these subgroups at several incidence angles, to derive on- and offaxis effective area, over the full NuSTAR bandpass.

Detailed PSF measurements will be carried out by removing the coarse aperture plate, thereby illuminating the entire azimuthal range of the individual subgroups, imaging it onto the EMCCD. Full flood PSF investigations will be carried out using both the Ge and the EMCCD detectors.

\section{RaMCaF Now and Tomorrow}

RaMCaF was inaugurated in late 2009 and early 2010, when it was used to validate the assembly process through X-ray testing the so-called process validation model 2 (PVM2). The test established the PSF and effective area of PVM2, a 10-layer NuSTAR flight optic prototype, directly out of the assembly room, then went on to confirm that a thermal vacuum bake, followed by a sine-burst shake test had no impact on its performance. See [3] for additional information on the test and its results.

Since then RaMCaF has been occupied testing prototype and flight multilayer-coated flat Silicon witness samples through a setup similar to the subaperture setup, compare for example, Figure 7(bottom).

Figure 10 compares results from $\mathrm{RaMCaF}$ to previous monochromatic measurements carried out at various synchrotron facilities around the world, demonstrating good agreement. The measured samples were flight-like coatings on flat Silicon coupons.

In late 2010 and early 2011, FM0 will be put through the baseline calibration approach to refine the plan for the next flight model, which is scheduled for calibration in March 2011. Facility performance [17] and initial results from the flight calibrations [18] will be presented in August 2011 at the SPIE Optics + Photonics in San Diego, CA.

Beyond the NuSTAR ground calibration, the facility will be used for a more detailed study of witness samples and potentially FM0. Further plans include turning RaMCaF into a user facility. With the ease-of-use and low maintenance mentality that it was built around, it is expected to play an important role in testing future X-ray optics and detectors while NuSTAR explores the hard X-ray Universe.

RaMCaF Note. James Rainwater, 1975 Nobel Laureate, served twice as director of the Nevis Laboratories during his tenure at Columbia University from 1946 until his retirement in 1986. He received his bachelor's degree in Physics from California Institute of Technology (Caltech) in 1939 and shared his Nobel Prize with Danish Aage Bohr and Danish-American Ben Roy Mottelson. An internal NuSTAR calibration facility naming contest selected the suggestion Rainwater Memorial Calibration Facility as the winning contribution due to the multitude of common points between James Rainwater's education and professional life and the NuSTAR collaboration, namely, the Technical University of Denmark, DTU (mirror coating), Columbia (optic PI), and Caltech (project and detector PI).

The NuSTAR mission, and through this RaMCaF, is funded by NASA through contract number NNG08FD60C. Additional contributions are provided by DTU.

Part of this work was performed under the auspices of the US Department of Energy by Lawrence Livermore National Laboratory under Contract DE-AC52-07NA27344.

\section{References}

[1] F. E. Christensen et al., "Graded d-spacing multilayer telescope for high-energy X-ray Astronomy," in Multilayer and Grazing Incidence X-Ray/EUV Optics, vol. 1546 of Proceedings of SPIE, pp. 160-168, 1991.

[2] F. A. Harrison et al., "The Nuclear Spectroscopic Telescope Array (NuSTAR)," in Space Telescopes and Instrumentation 2010: Ultraviolet to Gamma Ray Optics, vol. 7732 of Proceedings of SPIE, 2010.

[3] C. J. Hailey et al., "The Nuclear Spectroscopic Telescope Array (NuSTAR): optics overview and current status," in Space Telescopes and Instrumentation 2010: Ultraviolet to Gamma Ray Optics, vol. 7732 of Proceedings of SPIE, 2010.

[4] F. E. Christensen et al., "X-ray calibration of the SODART flight telescopes," in Grazing Incidence and Multilayer X-Ray Optical Systems, Proceedings of SPIE, pp. 294-306, 1997. 
[5] J. K. Koglin et al., "Calibration of HEFT hard X-ray optics," in Proceedings of the X-Ray Universe 2005, pp. 955-960, 2006.

[6] R. H. Redus, A. C. Huber, and D. J. Sperry, "Dead time correction in the DP5 digital pulse processor," in Proceedings of the 2008 IEEE Nuclear Science Symposium Conference Record (NSS/MIC '08), pp. 3416-3420, October 2008.

[7] October 2010, http://www.ni.com/labview/whatis/.

[8] S. Goto, K. Takeshita, Y. Suzuki et al., "Construction and commissioning of a 215-m-long beamline at SPring-8," Nuclear Instruments and Methods in Physics Research, Section A, vol. 467-468, pp. 682-685, 2001.

[9] F. E. Christensen, "High-resolution X-ray scatter and reflectivity study of sputtered Ir surfaces," in Multilayer and Grazing Incidence X-Ray/EUV Optics, vol. 2011 of Proceedings of SPIE, pp. 18-33, 1994.

[10] D. L. Windt, "IMD—Software for modeling the optical properties of multilayer films," Computers in Physics, vol. 12, no. 4, pp. 360-370, 1998.

[11] October 2010, http://www.ortec-online.com/.

[12] D. M. Schlosser, P. Lechner, G. Lutz et al., "Expanding the detection efficiency of silicon drift detectors," Nuclear Instruments and Methods in Physics Research, Section A, vol. 624, no. 2, pp. 270-276, 2010.

[13] S. C. Thacker et al., "Development of a high-speed CT imaging system using an EMCCD camera," in Physics of Medical Imaging, vol. 7258 of Proceedings of SPIE, 2009.

[14] T. Pantazis et al., "The historical development of the thermoelectrically cooled X-ray detector and its impact on the portable and hand-held XRF industries," in The Use and Application of Handheld and Portable XRF Spectrometers, vol. 39 of X-Ray Spectroscopy, pp. 90-97, 2009.

[15] October 2010, http://www.comet.ch.

[16] F. E. Christensen et al., "NuSTAR flight coatings: what did we really do," in Optics for EUV, X-Ray and Gamma-Ray Astronomy, vol. 8147 of Proceedings of SPIE, 2011.

[17] N. F. Brejnholt et al., "NuSTAR ground calibration: the Rainwater Memorial Calibration Facility (RaMCaF)," in Optics for EUV, X-Ray and Gamma-Ray Astronomy, vol. 8147 of Proceedings of SPIE, 2011.

[18] J. K. Koglin et al., "First results from the ground calibration of the NuSTAR flight optics," in Optics for EUV, X-Ray and Gamma-Ray Astronomy, vol. 8147 of Proceedings of SPIE, 2011. 